\title{
Gestational Methylazoxymethanol Acetate Treatment Impairs Select Cognitive Functions: Parallels to Schizophrenia
}

\author{
Robert E Featherstone ${ }^{*, 1}$, Zoe Rizos', José N Nobrega ${ }^{2,3,4}$, Shitij Kapur ${ }^{2,5}$ and Paul J Fletcher ${ }^{1,2,3}$ \\ 'Section of Biopsychology, Centre for Addiction and Mental Health, Toronto, ON, Canada; '2Department of Psychiatry, University of Toronto, \\ Toronto, ON, Canada; ${ }^{3}$ Department of Psychology, University of Toronto, Toronto, ON, Canada; ${ }^{4}$ Neuroimaging Research Section, Centre for \\ Addiction and Mental Health, Toronto, ON, Canada; ${ }^{5}$ Schizophrenia/PET Centre, Centre for Addiction and Mental Health, Toronto, ON, Canada
}

\begin{abstract}
Gestational methylazoxymethanol acetate (MAM) exposure has been suggested to produce neural and behavioral abnormalities similar to those seen in schizophrenia. In order to assess MAM treatment as a model of schizophrenia, pregnant female rats were injected with MAM $(22 \mathrm{mg} / \mathrm{kg})$ on gestational day 17 and their offspring were assessed in adulthood on a series of cognitive tasks. The first experiment involved an attentional set-shifting task, a rodent analog of the Wisconsin card sort task. In experiment 2, animals were tested on the 5-choice serial reaction time task, a rodent analog of the continuous performance task. In the final experiment animals were assessed on a differential reinforcement of low rate of responding $20 \mathrm{~s}$ schedule of reinforcement (DRL-20), a task that is sensitive to changes in inhibitory control. In the first experiment, MAM-treated animals required a greater number of trials than controls to successfully learn an extradimensional shift on the set-shifting task, and had difficulties in learning to reverse a previously acquired discrimination. In contrast, MAM-treated animals showed little impairment on the 5-choice task, aside from a modest but consistent increase in premature responding. Finally, MAM exposed animals showed substantial impairments in DRL performance. Post-mortem analysis of brain tissue showed significant decreases in tissue weight in the hippocampus, parietal cortex, prefrontal cortex, and dorsal striatum of MAM-treated animals. These results support the notion that MAM treatment may simulate some aspects of schizophrenic cognition. Neuropsychopharmacology (2007) 32, 483-492. doi: I 0. I038/sj.npp. I 30 I223; published online I I October 2006
\end{abstract}

Keywords: attentional-set shifting; 5-choice serial reaction time; DRL; prefrontal cortex; extra-dimensional shift; reversal learning

\section{INTRODUCTION}

Neonatal methylazoxymethanol acetate (MAM) treatment induces brain abnormalities that may be similar to the types of changes seen in schizophrenia, including abnormalities in hippocampal (Chen and Hillman, 1986; Ferguson and Holson, 1997; Flagstad et al, 2004; Gourevitch et al, 2004; Shimizu et al, 1991; Virgili et al, 1997) and cortical (Lavin et al, 2005; Talamini et al, 1999) function and morphology. These changes are accompanied by changes in mesolimbic dopamine function, as indicated by an increased sensitivity to the behavioral and neurochemical effects of amphetamine (Ferguson and Holson, 1997; Flagstad et al, 2004). Animals exposed to MAM during gestation show impairments within cognitive domains known to be disrupted in schizophrenia, such as attention (Flagstad et al, 2005; Mohammed et al, 1986a, b; Talamini et al, 2000), long-term memory (Fiore et al, 2001, 2002; Gourevitch $e t$ al, 2004; Lee and Rabe, 1992; Shimizu et al, 1991), working

\footnotetext{
*Correspondence: Dr RE Featherstone, Section of Biopsychology, Centre for Addiction and Mental Health, 250 College Street, Toronto, ON, Canada M5T IR8, Tel: + I 416535850 I (ext 6242), Fax: + I 416979 6942, E-mail: Rob_Featherstone@camh.net

Received 2I March 2006; revised I 4 June 2006; accepted 12 July 2006 Online publication: 12 September 2006 at http://www.acnp.org/ citations/Npp09 | 206060 I 82/default.pdf
}

memory (Gourevitch et al, 2004), and behavioral flexibility (Fiore et al, 2002; Flagstad et al, 2005; Leng et al, 2005), although negative findings have also been reported in many of these domains (Flagstad et al, 2005; Jongen-Relo et al, 2004). As such, prenatal methylazoxymethanol treatment has recently attracted attention as a potential animal model of schizophrenia (Grace and Moore, 1998; Moore et al, 2006).

While the precise timing of MAM administration differs across studies, in some cases occurring as early as gestational day 9 (Jongen-Relo et al, 2004; Talamini et al, 2000) and as late as the postnatal period ( $\mathrm{Lu}$ et al, 2000), there is a growing consensus that MAM treatment at gestational day 17 is the optimal strategy for producing schizophrenia-like changes. Treatment occurring on or before gestational day 15 produces widespread disruptions of brain morphology and behavior (Gourevitch et al, 2004) and it is likely that these changes are too nonspecific to serve as a useful model of schizophrenia. Treatment at gestational day 17 produces much less intense changes in brain and behavior (Gourevitch et al, 2004), and treatment at this time produces deficits within many cognitive domains known to be impaired in schizophrenia (Flagstad et al, 2004, 2005; Gourevitch et al, 2004; Moore et al, 2006).

As part of an effort to develop and assess novel treatments for cognitive deficits in schizophrenia, researchers have 
begun an attempt to delineate the cognitive domains in which schizophrenics are impaired and to develop a standardized test battery to measure performance in these domains (Green et al, 2004). Some researchers have suggested adopting a similar approach for animal studies (Nuechterlein et al, 2005), with emphasis placed on finding tasks that can serve as equivalent versions of those tasks selected for use in humans. Two tasks that have been singled out as promising candidates in this regard are the attentional set-shifting task (Birrell and Brown, 2000) and the 5-choice serial reaction time task. In the set-shifting task rats are required to solve a series of discriminations by attending to a particular perceptual dimension of a multidimensional stimulus. A critical discrimination occurs when rats are required to shift to an alternate perceptual dimension after having acquired an attentional set to the previous dimension. The extradimensional shift (EDS) required to solve this discrimination is analogous to what is required to solve the Wisconsin Card Sort Task, a test that has repeatedly shown impaired prefrontal mediated executive function in schizophrenia (Goldberg et al, 1987). The rodent set-shifting task also requires the prefrontal cortex (Birrell and Brown, 2000; McAlonan and Brown, 2003) and, thus, is likely a useful test for assessing cognition in a putative animal model of schizophrenia. The 5-choice serial reaction time task, which measures sustained attention, was developed to serve as a rodent version of the continuous performance task (Robbins, 2002). Schizophrenic patients typically display deficits in sustained attention and these deficits often precede the onset of psychotic symptoms by several years (Chen and Faraone, 2000). In the 5-choice task, animals are required to attend to brief presentations of a light stimulus that occur in one of five locations. Owing to the unpredictability of the stimulus, animals are required to focus attention across each of the five potential locations. Performance of the 5-choice task depends on a number of brain areas, including the prefrontal cortex (Christakou et al, 2001; Chudasama et al, 2003; Muir et al, 1996; Passetti et al, 2002) and dorsal striatum (Brown and Robbins, 1989; Rogers et al, 2001).

In the present experiment, pregnant females were treated with MAM $(22 \mathrm{mg} / \mathrm{kg})$ or saline on day 17 of pregnancy. Offspring from these animals were assessed in adulthood on an attentional set-shifting task, followed by the 5-choice serial reaction time task. As preliminary results suggested poor inhibitory response control in MAM-treated animals, the study was extended to assess performance on a differential reinforcement of a low rate of response (DRL) schedule. Optimal performance on this task requires that animals learn to withhold responding for short periods of time $(20 \mathrm{~s})$ in order to reliably receive reinforcement. This constitutes the first report examining attentional set-shifting and sustained attention in the MAM model.

\section{METHODS}

\section{Subjects}

Timed pregnant Sprague-Dawley rats arrived on day 10 of pregnancy from Charles Rivers Laboratories (SaintConstant, QC). These rats were injected with either saline $(n=3)$ or MAM $(22 \mathrm{mg} / \mathrm{kg}$, in a volume of $5 \mathrm{mg} / \mathrm{ml}$,
Midwest Research Institute, Kansas City, MO) $(n=8)$ on day 17 of pregnancy (day 0 defined as the day of conception). Mean litter size in saline-treated mothers was 13.3 pups, while mean litter size in MAM-treated mothers was 11.6 pups. Eleven saline-treated and 13 MAM-treated animals were selected for the current experiment, with each group consisting at least one animal from each litter. On day 28, pups were sexed and were separated from the mother, at which time they were pair housed with a sibling in a clear plastic cage $(48 \times 27 \times 20 \mathrm{~cm})$. Only male rats were used. Rats were singly housed beginning roughly 2 weeks before behavioral training (around 80 days). Housing rooms were maintained on a 12:12 light/dark schedule (0800-2000 hours). During testing, rats were food restricted to approximately $85 \%$ of their body weight. All procedures were carried out in accordance with the Canadian Council on Animal Care and the institutional animal care committee, as well as the National Institute of Health Guide for the Care and Use of Laboratory Animals. All efforts were made to minimize the number of animals used and their suffering. This project was performed, in part using compound(s) provided by the National Cancer Institute's Chemical Carcinogen Reference Standards Repository operate under contract by Midwest Research Institute, NO. N02-CB-07008.

\section{Experiment 1: Attentional Set-Shifting}

Apparatus. Training took place in a black Plexiglas chamber $(60 \mathrm{~cm}$ long by $42 \mathrm{~cm}$ wide by $30 \mathrm{~cm}$ tall). The front of the chamber was divided into two separate compartments, each of which measured $21.5 \mathrm{~cm}$ long by $20.5 \mathrm{~cm}$ wide. A moveable opaque barrier was used to block access to the front of the chamber. At the beginning of each trial, the animal was placed in the back of the chamber, with the barrier blocking access to the front. When the barrier was raised, animals had access to either compartment. A small ceramic bowl $(7 \mathrm{~cm}$ in diameter and $4 \mathrm{~cm}$ in depth) was placed in each compartment. Each bowl was covered with a texture (fine sandpaper, course sandpaper, velvet, the reversed side of the velvet material, paper, or plastic wrap), and was filled with bedding material (Bed-O'cobs, Andersonsinc, Maumee, OH). The bedding material was scented with an odor (paprika, thyme, cinnamon, cumin, cloves, or sage).

Procedure. The procedure for set-shifting was similar to that used by Birrell and Brown (2000) and Fletcher et al (2005). Animals were initially trained to dig for food reward. Two bowls were placed in the chamber, each containing a small amount of bedding and a half piece of Froot-loop cereal. Initially, the cereal was located on top of the bedding, but, over successive trials, was placed deeper within the bedding such that the rat would have to dig to retrieve it. Training continued until animals were able to reliably (six trials in a row) dig and retrieve food from each bowl. Animals were then trained on a simple discrimination (SD), in which food was paired with one stimulus dimension (either odor or texture) but not the other. The stimuli during this discrimination were banana and vanilla or masking tape and paper towel. Training continued until the animals chose correctly over six consecutive trials.

The next phase of training, which took place on the following day, involved the full set of discriminations. Two 
bowls were placed in the apparatus, with only one bowl baited with a half-piece of Froot-loop. The location of the correct bowl was randomly determined on every trial to prevent the development of a positional bias. The bowls differed according to odor and texture, with food being consistently associated with a particular odor or texture depending on the current discrimination. Discrimination training began with a SD. For this discrimination, only one stimulus dimension was present. In the second discrimination (CD), another stimulus dimension was added, although food remained paired with the original stimulus. The third discrimination involved a reversal of the second discrimination, in which an alternate exemplar of the relevant stimulus dimension was now paired with food. The fourth discrimination required animals to choose between a new series of stimuli, with the previously relevant stimulus dimension still paired with food (intradimensional shift (IDS)). This discrimination was followed by a reversal in which the alternate exemplar of the relevant stimulus dimension was now paired with food. In the sixth discrimination (EDS) the relevant stimulus dimension was changed, such that the animal had to learn to attend to the previously irrelevant dimension. The final discrimination involved a reversal of the EDS discrimination in which the animal had to choose the previously irrelevant exemplar of the new stimulus dimension. The full series of discriminations is outlined in Table 1. Six saline-treated and seven MAM-treated animals received initial training (SD, CD, Rev 1 , and IDS) with odor as the relevant dimension, and were required to make an EDS to texture. Five saline and six MAM-treated animals received initial training with texture as the relevant dimension, followed by a shift to odor during the EDS. A correct trial was considered to have occurred when the animal successfully retrieved the Froot-Loop from the bowl. If the animal dug in the incorrect bowl, the trial was scored as an error. On the first four trials, an incorrect response was simply recorded and the trial continued until the animal successfully retrieved the Froot-loop piece from the correct bowl. Beginning on the fifth trial, incorrect responses caused the trial to be terminated. Animals were moved onto the next discrimination once they had made six correct responses in a row. The individual who carried out the set-shifting task was unaware of the group identity of any given animal.

\section{Experiment 2: 5-Choice Serial Reaction Time Task}

Apparatus. The 5-choice serial reaction time task took place in eight operant chambers (Med Associates, St Albans, VT). Chambers were equipped with a magazine/liquid dipper, located on one wall, and five apertures each containing an LED located on the opposite wall. The magazine and light apertures contained photodetectors to detect nose poke entries. Events inside the chamber were controlled by Med Associates equipment and software, using an in-house program written in MED-PC. A $10 \%$ sucrose/water solution $(0.06 \mathrm{ml}$ per reinforcement) served as reinforcement on the task.

Procedure. Training began 5 days following the set-shifting experiment. Animals were initially trained to respond to signaled presentations of the dipper. Each session began
Table I Example of Training Procedure for the Attentional Set-Shifting Task

\begin{tabular}{|c|c|c|}
\hline Stage & Exemplar & Combinations \\
\hline Simple (SD) & $\mathrm{Ol+}$ & $\mathrm{O} 2-$ \\
\hline \multirow[t]{2}{*}{ Compound (CD) } & $\mathrm{OI} / \mathrm{TI}+$ & $\mathrm{O} 2 / \mathrm{T} 2-$ \\
\hline & $\mathrm{Ol} / \mathrm{T} 2+$ & $\mathrm{O} 2 / \mathrm{TI}-$ \\
\hline \multirow[t]{2}{*}{ Reversal I } & $\mathrm{O} 2 / \mathrm{T} 2+$ & $\mathrm{OI} / \mathrm{TI}-$ \\
\hline & $\mathrm{O} 2 / \mathrm{TI}+$ & Ol/T2- \\
\hline \multirow[t]{2}{*}{ Intradimensional shift } & O3/T3+ & O4/T4- \\
\hline & O3/T4+ & O4/T3- \\
\hline \multirow[t]{2}{*}{ Reversal 2} & O4/T3+ & O3/T4- \\
\hline & O4/T4+ & O3/T3- \\
\hline \multirow[t]{2}{*}{ Extradimensional shift } & $\mathrm{T} 5 / \mathrm{O} 5+$ & $\mathrm{T} 6 / \mathrm{O6}-$ \\
\hline & T5/O6+ & T6/O5- \\
\hline \multirow[t]{2}{*}{ Reversal 3} & $\mathrm{~T} 6 / \mathrm{O} 5+$ & $\mathrm{T} 5 / \mathrm{O6}-$ \\
\hline & $\mathrm{T} 6 / 06+$ & $\mathrm{T} 5 / \mathrm{O} 5-$ \\
\hline
\end{tabular}

Example of the procedure followed for the attentional set-shifting task. The left column depicts stage of training (type of discrimination), while the middle column depicts the exemplar combinations used for that stage of training, and the right column indicates the particular stimuli used. $O$ and $T$ refer to odor and texture, respectively, and the plus and minus signs indicate which exemplar combination was paired with reward $(+)$ or no reward $(-)$. In this particular case, odor was initially the relevant dimension and the extradimensional shift (EDS) involved a shift to texture. This was counterbalanced such that some animals received texture first and had to make an EDS to odor

with the dipper raised and the magazine light illuminated. Once the animal had made a nose poke into the magazine the light was extinguished and the dipper lowered. This procedure was repeated every $5 \mathrm{~s}$. Each session lasted for 30 min or until 60 responses had been made. Subjects were given three sessions of this training, which was sufficient for all animals to meet the response criterion of 60 responses during at least one session.

Immediately following magazine training, animals began the 5-choice task. Each session lasted for 30 min or until 100 trials had been completed. To initiate a trial, the subject was required to make a nose poke response in the illuminated magazine. Five seconds following a magazine response, one of the five nose poke apertures was illuminated. A nose poke response to the lit aperture (either during light onset or during a $5 \mathrm{~s}$ limited hold period following termination of the light) extinguished the light and caused the liquid dipper to be raised for a $3 \mathrm{~s}$ period, while a nose poke response to a nonlit aperture caused the light to be extinguished followed by a $5 \mathrm{~s}$ time out. Nose poke responses that occurred during the $5 \mathrm{~s}$ between initiation of the trial and illumination of the nose poke aperture were recorded as premature responses, and caused the trial to be terminated, followed by a $5 \mathrm{~s}$ time out. Finally, a failure to make a nose poke response to the lit aperture was recorded 
as an omission, and led to a $5 \mathrm{~s}$ time out. During training, stimulus durations (the amount of time that nose poke apertures were illuminated) were reduced as performance improved. Animals were initially trained at a duration of $30 \mathrm{~s}$, and remained at this level until their performance had surpassed a prespecified criterion, at which point they were shifted to the next lowest duration $(30,20,10,5,2.5,1.25$, and $1 \mathrm{~s})$. The criterion for each stage was as follows. At 30 and $20 \mathrm{~s}$, animals were required to perform at least 30 (out of 100) correct responses. At $10 \mathrm{~s}$, this was increased to 50 correct trials. For 5, 2.5, 1.25, and $1 \mathrm{~s}$, animals were required to make 50 or more correct responses, have greater than $80 \%$ accuracy and less than $20 \%$ omitted trials. Animals were trained for a total of 41 sessions in order to reach criterion at the lowest stimulus duration of $1 \mathrm{~s}$.

Once stable performance had been achieved at the $1 \mathrm{~s}$ stimulus duration animals were given three additional tests. First, animals were challenged with different stimulus durations $(0.125,0.25,0.5$, and $1 \mathrm{~s})$. This was performed using a within-subject design, with each test day separated by a maintenance session using a $1 \mathrm{~s}$ stimulus duration. The purpose of this manipulation was to increase demand on attentional resources. Second, animals were given an additional session using a prolonged ITI ( $9 \mathrm{~s}$ instead of $5 \mathrm{~s}$ ). This manipulation typically increases premature responding, since animals must delay responding across a longer duration. Finally, animals were given a session of training with variable ITIs $(3.5,5.5,7.5$, and $9.5 \mathrm{~s})$ occurring within the same session.

\section{Experiment 3: Differential Reinforcement of a Low Rate of Response}

Apparatus. DRL training took place in standard operant chambers (Med Associates, St Albans, VT). Each chamber contained a response lever (located left of the magazine), a pellet dispenser, and a houselight. Events inside the chamber were controlled by Med Associates equipment and software. Standard $45 \mathrm{mg}$ food pellets (Bioserve, Frenchtown, NJ, USA) were used as reinforcement.

Procedure. Training on the DRL task began 5 days following the end of 5-choice training.

Initially, three sessions of continuous reinforcement bar press training were given. Each session lasted for $30 \mathrm{~min}$, or until a total of 100 responses had been made. In order to be considered ready for DRL training, animals were required to make 100 responses over each of two consecutive sessions. DRL training was identical to bar press training, with the exception that reinforcement was now delivered according to a DRL-20 schedule of reinforcement. The first response of each session was always reinforced and served as a start point for the interval timer. To receive additional reinforcement animals were required to withhold responding for an interval of at least $20 \mathrm{~s}$. Responses occurring earlier than $20 \mathrm{~s}$ reset the interval timer. Each session lasted for approximately $40 \mathrm{~min}$.

Post-mortem analysis. Subjects were killed by rapid decapitation. The brain was removed in less than a minute and rinsed in ice-cold normal saline, blotted, then dissected on a thermoelectric cold plate. The brain was separated into rostral (RP) and caudal (CP) pieces by a coronal cut $1 \mathrm{~mm}$ $\mathrm{RP}$ to the CP edge of the olfactory tubercle. Bilateral structures were removed and combined. Nucleus accumbens was identified in the cut surface of the RP and removed. A second coronal cut $3 \mathrm{~mm} \mathrm{CP}$ to the anterior pole of the RP removed the prefrontal cortex whereupon the medial $1 / 4$ was harvested as medial prefrontal cortex. The cerebellum was removed from the CP. Shallow cuts placed in the surface of the cerebral cortex (CC) outlined the occipital and parietal cortex for later removal. The amygdala was removed in block of tissue from the basal surface of the CP outlined by sagittal cuts in the temporal cortex down to the rhinal fissure (rf) at the level of the optic chiasm and at the CP edge of the hypothalamus (HY). A $2 \mathrm{~mm}$ deep stab incision along the $\mathrm{rf}$ between these previous cuts allowed the block to be separated at the medial border of the HY. Vertical stab incisions $3 \mathrm{~mm}$ deep around the periphery of the HY allowed for its removal. The CCs were separated by a sagittal cut through the corpus callosum then carefully retracted medially to expose hippocampus (HI) and RP ST. The HIs were separated at the midline then removed. The CP ST was first separated from the thalamus (TH) along the internal capsule then removed using the external capsule as a dissection plane. The RP STs in the $\mathrm{RP}$ were also taken. The CCs were then divided along the previous cuts into occipital and parietal portions. The temporal and paleocortex were removed from the diencephalon as was the septum. The $\mathrm{TH}$ was removed by a coronal cut RP to the superior geniculate bodies. The midbrain was separated from the pons/medulla with a coronal cut tilted CP to follow the CP edge of the inferior geniculate bodies. Each harvested area was placed in a micro test-tube labeled with area and subject number then frozen on dry ice and stored in a $-80^{\circ} \mathrm{C}$ freezer for later tissue weight determinations.

\section{RESULTS}

\section{Attentional Set-Shifting}

Thirteen MAM and 11 saline-treated animals were used. Figure 1 depicts performance on the set-shifting task. A repeated measures ANOVA was conducted on the number of trials to criterion, using group as a between-subject variable and discrimination type as a within-subject variable. A significant main effect was found for group $(\mathrm{F}(1,22)=6.99, p=0.015)$, discrimination type $(\mathrm{F}(6,132)=$ 185.39, $p=0.0001)$ and for the group $\times$ discrimination type interaction term $(\mathrm{F}(6,132)=9.07, p=0.0001)$. Planned comparison analyses were then conducted which compared the two groups during each stage of training. MAM-treated animals required a significantly greater number of trials to reach criterion during the EDS discrimination $(\mathrm{F}(1,22)=14.53, p=0.001)$. A similar pattern was observed during two of the three reversal discriminations (Rev 1: $\mathrm{F}(1,22)=7.78, \quad p=0.011$ and Rev 3: $\mathrm{F}(1,22)=10.98$, $p=0.003$ ), with MAM animals requiring more trials to reach criterion. No group differences were observed during any of the other discriminations. Additionally, Levene's test for equality of variance showed equivalent amounts of variance in the two groups. 


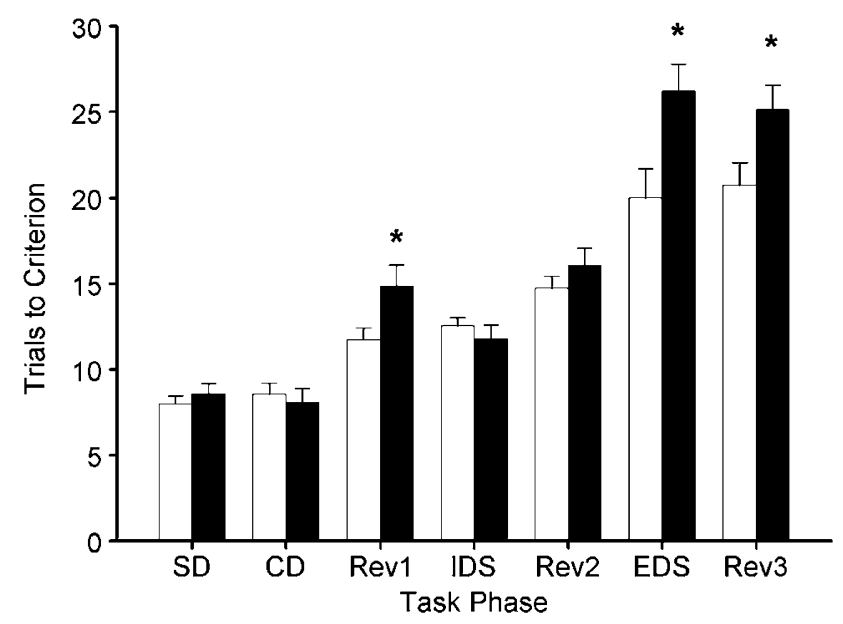

Figure I Mean ( + SEM) number of trials to reach criterion on the attentional set-shifting task in rats treated MAM $(n=13)$ (black bar) or saline $(n=11)$ (white bar). Asterisk indicates statistical significance $(p<0.05)$. MAM-treated animals were impaired on discriminations requiring a reversal (Rev I and Rev 3) or an EDS (EDS), but were similar to saline animals in performance of other discriminations (SD $=$ simple discrimination; $C D=$ compound discrimination; $I D S=$ intra-dimensional shift).

\section{5-Choice Serial Reaction Time}

Two animals (one MAM- and one saline-treated animal) were removed due to consistently poor performance. Thus, 12 MAM-treated and 10 saline-treated animals were used. Animals reached criterion after one session of training at each of the first five stimulus durations $(30,20,10,5$, and $2.5 \mathrm{~s}$ ). Lowering the stimulus duration from 2.5 to $1.25 \mathrm{~s}$ greatly impacted performance of the task in animals and required that training schedules be tailored to the performance of each animal. This made it difficult to collect data that could be analyzed in a meaningful way. However, all animals had reached criterion by the end of training (41 days in total) with no obvious group differences on any of the performance variables (data not shown).

Data from the postacquisition manipulations (shortened stimulus duration test, variable ITI test and lengthened ITI test) served as the sole analysis of performance. All analyses assessed percent accuracy, percentage of omitted trials, number of perseverative responses, latency (in seconds) to perform the correct response, latency (in seconds) to collect reinforcement, and number of premature responses. Each analysis involved a repeated measure ANOVA, using group as a between-subject variable and stimulus duration (or ITI) as a within-subject variable. Additionally, each analysis included a test for homogeneity of variance (Levene's test for equality of variance). With the exception of premature responding during the $1 \mathrm{~s}$ stimulus duration test, group variance was found to be homogeneous at all times.

After acquisition of the task, performance was assessed at a number of different stimulus durations $(1,0.5,0.25$, and $0.125 \mathrm{~s}$ ). For correct latency, reinforcement latency, percent accuracy, and percent omission, no significant differences were observed, save for a significant effect of stimulus duration $\quad((\mathrm{F}(3,60)=6.23, \quad p<0.05) ; \quad(\mathrm{F}(3,60)=6.14$, $p<0.05) ;(\mathrm{F}(3,60)=133.38, p<0.05)$ and $(\mathrm{F}(3,60)=35.73$,
Table 2 Effects of MAM on 5-Choice Performance with a Shortened Stimulus Duration

\begin{tabular}{|c|c|c|c|c|c|}
\hline \multirow[b]{2}{*}{ Measure } & \multirow[b]{2}{*}{ Group } & \multicolumn{4}{|c|}{ Stimulus duration (s) } \\
\hline & & I & 0.5 & 0.25 & 0.125 \\
\hline \multirow[t]{2}{*}{ (A) Accuracy } & SAL & $91.2(1.3)$ & $74.3(3.5)$ & $62.9(3.6)$ & $46.0(3.1)$ \\
\hline & MAM & $86.4(2.0)$ & $77.7(3.3)$ & $58.4(2.5)$ & $49.7(3.6)$ \\
\hline \multirow[t]{2}{*}{ (B) Premature } & SAL & $8.6(3.0)$ & $6.3(2.2)$ & $10.3(4.04)$ & $6.0(1.5)$ \\
\hline & MAM & $7.6(1.4)$ & $8.4(1.5)$ & $10.3(2.92)$ & | $4.6(3.4)$ \\
\hline \multirow[t]{2}{*}{ (C) Omission } & SAL & $9.7(1.9)$ & $25.3(4.9)$ & $36.7(6.2)$ & $43.8(6.6)$ \\
\hline & MAM & $6.8(1.9)$ & I5.3 (6.2) & $25.6(6.2)$ & $31.4(5.4)$ \\
\hline \multirow[t]{2}{*}{ (D) Perseverative } & SAL & $7.3(1.3)$ & $3.8(1.1)$ & $1.8(0.5)$ & $1.0(0.4)$ \\
\hline & MAM & $5.7(0.8)$ & $3.4(0.5)$ & $2.5(0.6)$ & $3.3(0.5)$ \\
\hline \multirow[t]{2}{*}{ (E) Correct lat } & SAL & $0.98(0.16)$ & $0.78(0.09)$ & $0.69(0.06)$ & $0.66(0.05)$ \\
\hline & MAM & $0.87(0.08)$ & $0.75(0.11)$ & $0.59(0.04)$ & $0.61(0.03)$ \\
\hline \multirow[t]{2}{*}{ (F) Reinforce lat } & SAL & $0.9(0.02)$ & $0.93(0.02)$ & $0.94(0.03)$ & $0.98(0.03)$ \\
\hline & MAM & $0.99(0.16)$ & $1.02(0.03)$ & $1.06(0.05)$ & $1.03(0.04)$ \\
\hline
\end{tabular}

Following acquisition of the 5-choice task, animals were assessed at a series of shortened stimulus durations $(0.5,0.25$, and $0.125 \mathrm{~s})$ in MAM- and saline-treated animals. The table shows mean (+ SEM) percent accuracy (A), premature responses $(B)$, percentage of omitted trials $(C)$, and number of perseverative responses (D) latency to respond on trials with a correct response $(E)$ and latency to collect reward (F) in MAM- vs saline-treated animals. The only significant result was the interaction between group and stimulus duration for perseverative responses.

$p<0.05$ ), respectively). For premature responding, no significance was observed for either main effect or interaction, although there was a trend towards a significant group $\times$ stimulus duration interaction $(p=0.07)$. For perseverative responding, a significant effect was found for stimulus duration $(\mathrm{F}(3,60)=20.9, p<0.05)$ as well as for the group by stimulus duration interaction $(\mathrm{F}(3,60)=3.25$, $p<0.05)$. A Newman-Keuls post hoc test suggested that the significant interaction was likely due to a greater degree of change over the range of stimulus durations in salinetreated animals, although this result remains somewhat unclear as this data failed the test for homogeneity of variance. No significance was found for either latency measure. Table 2 shows these data. The failure to find a significant difference at the $1 \mathrm{~s}$ stimulus duration strongly suggests that animals in the two groups had acquired the task equally well before undergoing this manipulation.

After completing the stimulus duration test, animals were given a session with a lengthened ITI ( $9 \mathrm{~s})$. A $t$-test was used to analyze these data, with separate tests being conducted for each measure. No significant differences were found for any of the measures. See Table 3 for details.

In the last stage of testing, animals were given a session with variable ITIs (3.5, 5.5, 7.5, and $9.5 \mathrm{~s}$ ). For accuracy, premature responding and omission, significant main effects were found for ITI $((\mathrm{F}(3,60)=5.27, p<0.05)$, 
$(\mathrm{F}(3,60)=19.26, p<0.05)$ and $(\mathrm{F}(3,60)=2.82, p<0.05)$ respectively), while group and the group by ITI interaction were not significant, although a trend towards significance for the group by ITI interaction $(p=0.10)$ was found for premature responses. No significance was observed for either latency measure. See Table 4 for details.

\section{Differential Reinforcement of a Low Rate of Response}

A total of 13 MAM and 10 saline-treated animals were run on this task (the MAM-treated animal removed from the previous 5-choice task was run on this task). Training on the DRL task took place over 24 sessions, with data

Table 3 Effects of MAM on 5-Choice Performance with a Lengthened ITI

\begin{tabular}{lcc}
\hline Measure & SAL & MAM \\
\hline (A) Accuracy & $80.7(4.1)$ & $70.6(6.9)$ \\
(B) Premature & $29.1(6.4)$ & $51.2(9.7)$ \\
(C) Omission & $36.5(4.6)$ & $32.3(7.6)$ \\
(D) Perseverative & $3.2(1.0)$ & $3.0(0.7)$ \\
(E) Correct lat & $0.79(0.05)$ & $0.73(0.08)$ \\
(F) Reinforce lat & $0.95(0.03)$ & $0.93(0.09)$ \\
\hline
\end{tabular}

Mean (+SEM) percent Accuracy (A), premature responses (B), number of omitted trials $(C)$, perseverative responses $(D)$, latency to respond on trials with a correct response (E), and latency to collect reward (F) in MAM- vs salinetreated animals during a session with a lengthened intertrial interval (ITI). No significant differences were found between the two groups on any of these measures.

Table 4 Effects of MAM on 5-Choice Performance with Variable ITls

\begin{tabular}{lccccc}
\hline & & \multicolumn{5}{c}{ ITI Length (s) } \\
\cline { 3 - 6 } Measure & Group & $\mathbf{3 . 5}$ & $\mathbf{5 . 5}$ & $\mathbf{7 . 5}$ & $\mathbf{9 . 5}$ \\
\hline (A) Accuracy & SAL & $89.1(3.9)$ & $80.2(5.6)$ & $72.2(5.8)$ & $77.1(6.2)$ \\
& MAM & $88.9(3.8)$ & $83.1(3.8)$ & $75.4(4.0)$ & $76.7(6.1)$ \\
& & & & & \\
(B) Premature & SAL & $0.7(0.3)$ & $2.1(0.9)$ & $9.2(4.8)$ & $11.8(4.5)$ \\
& MAM & $1.4(0.5)$ & $2.0(0.5)$ & $8.5(2.5)$ & $21.3(4.4)$ \\
& & & & & \\
(C) Omission & SAL & $21.2(5.6)$ & $18.2(2.5)$ & $18.7(4.0)$ & $26.4(6.1)$ \\
& MAM & $19.0(4.1)$ & $15.8(3.4)$ & $18.6(4.3)$ & $26.0(7.2)$ \\
\hline
\end{tabular}

Mean (+SEM) percent accuracy (A), premature responses (B), number of omitted trials (C), in MAM- vs saline-treated animals during a session with a variable intertrial interval (ITI). Overall, no significant differences were observed between MAM and saline animals in regards to accuracy, omission, or premature responses. collapsed into 8, 3-day blocks. Four measures were recorded; number of reinforcers earned per session, number of responses per session, efficiency (number of responses per session divided by the number of reinforcers per session, multiplied by 100), and mean inter-response time (M-IRT) (mean interval between responses, excluding responses separated by an interval less than $2 \mathrm{~s}$ ). Separate repeated measures ANOVAs were conducted on each of these measures, with each analysis using group as a between-subject variable and block as a within-subject repeated variable. With the exception of number of responses, each measure showed some degree of inequality in regards to variance between the two groups. Specifically, for number of reinforcers earned this occurred during block 2 , for efficiency this occurred during blocks 1 and 2 and for M-IRT this occurred during blocks 1 and 2. To compensate for this, alpha was set at 0.01 for all analyses. For number of reinforcers earned, a significant effect was found for group $(\mathrm{F}(1,21)=9.54, p<0.01)$, block $(\mathrm{F}(7,147)=17.16, p<0.01)$, and the group by block interaction $(\mathrm{F}(7,147)=4.2$, $p<0.01)$. A Newman-Keuls post hoc test showed that MAM-treated animals earned significantly fewer reinforcers during every block except the first and third $(p<0.01)$ (see Figure 2a). Similarly, for number of responses, significant effects were found for block $(F(7,147)=98.3, p<0.01)$, and the group by block interaction term $(F(7,147)=3.6$, $p<0.01)$. A Newman-Keuls post hoc test was carried out on these data, which showed a significantly higher number of responses for MAM-treated animals during blocks 1-3 $(p<0.01)$ and during blocks 6 and $8 \quad(p<0.01)$ (see Figure $2 \mathrm{~b}$ ). For efficiency, a significant main effect was found for block $(\mathrm{F}(7,147)=22.82, p<0.01)$ and the group by block interaction $(F(7,147)=4.34, p<0.01)$, suggesting that only saline-treated animals showed an improvement in performance across the duration of testing (see Figure 2c). A Newman-Keuls post hoc test showed significantly poorer efficiency in MAM-treated animals during blocks 2, 6, 7, and $8(p=0.01)$. Finally, mean IRT was also significant for both group $(\mathrm{F}(1,21)=11.13, p<0.01)$ and block $(\mathrm{F}(7,147)=9.10, p<0.01)$ and the group by block interaction $(F(7,147)=3.79, p<0.01)$. Significant differences were found between MAM- and saline-treated animals during blocks $1,6,7$, and $8(p<0.01)$, with MAM-treated animals showing a significantly lower mean IRT than saline-treated animals (see Figure $2 \mathrm{~d}$ ).

\section{Post-Mortem Analysis}

Separate one-way ANOVAs were conducted for each individual brain area, with group serving as the independent variable and brain weight serving as the dependent variable. MAM-treated animals showed significantly reduced tissue weight in the $\mathrm{HI}(\mathrm{F}(1,23)=34.34, p<0.05)$, parietal cortex $(\mathrm{F}(1,23)=28.70, p<0.05)$, medial prefrontal cortex $(\mathrm{F}(1,23)=8.19, p<0.05)$, orbital prefrontal cortex $(\mathrm{F}(1,23)=6.20, p<0.05)$, and dorsal ST $(\mathrm{F}(1,23)=6.12$, $p<0.05)$. Additionally, MAM-treated animals showed significantly greater weight for the cerebellum $(\mathrm{F}(1,23)=4.87$, $p<0.05)$. Brain weight did not differ in any of the other brain areas. Brain weight data are shown in Table 5. 

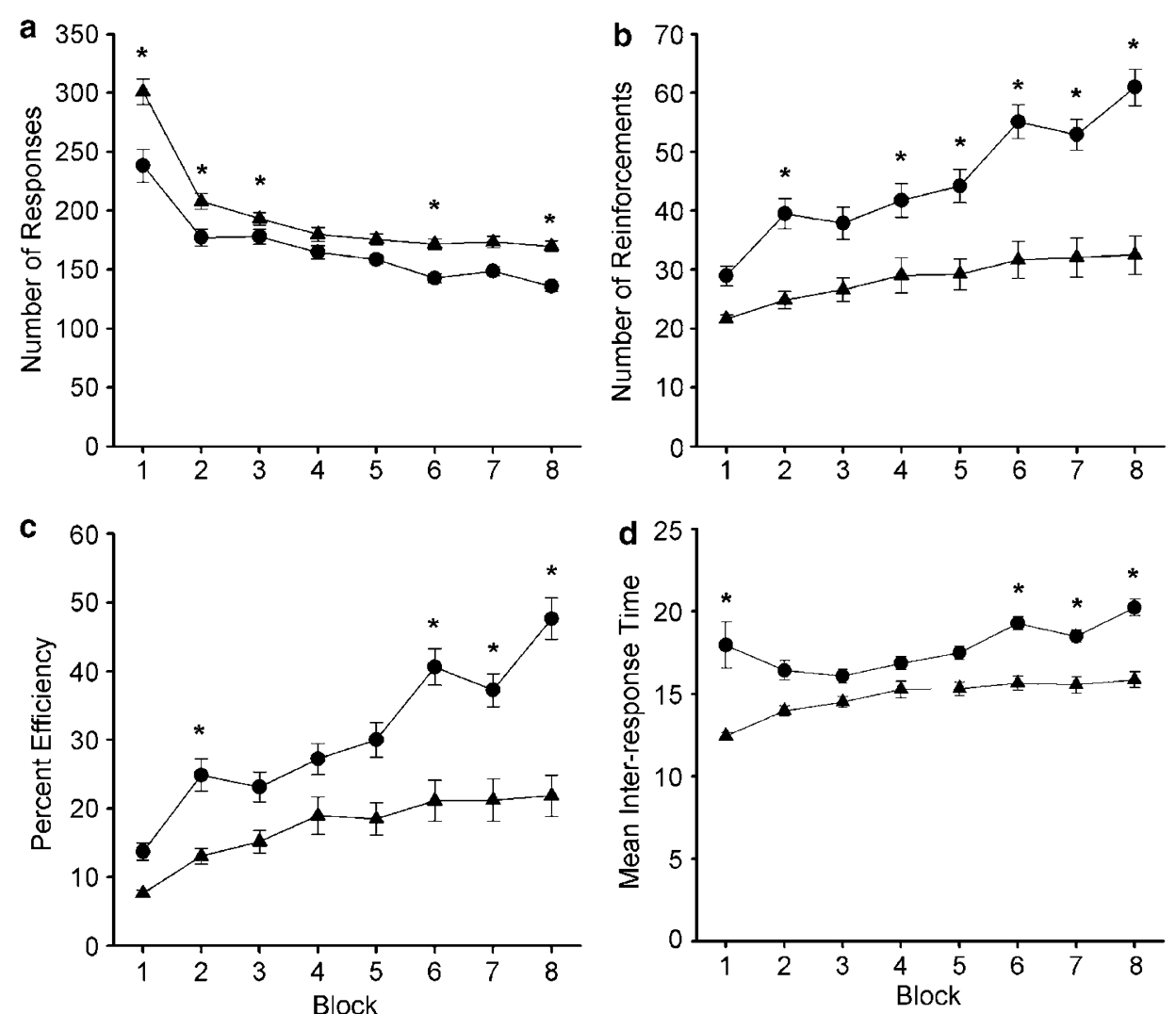

Figure 2 Mean (+SEM) (a) number of reinforcers earned, (b) number of responses performed, (c) efficiency, and (d) M-IRT during training on a DRL in MAM-treated (MAM) $(-\boldsymbol{\Delta}$-) and saline-treated (SAL) (-) animals. Each block represents three sessions of training. Asterisk indicates significant ( $p<0.05)$ differences. Significant differences were observed between MAM-treated and saline-treated animals on each measure.

\section{DISCUSSION}

On the attentional set-shifting task, compared to controls, MAM-treated animals required a greater number of trials to learn the EDS component of the task, indicative of a deficit in shifting attentional set. MAM-treated rats were able to solve a SD, and perform an IDS, suggesting that the deficit in EDS ability was not due to a generalized performance or cognitive impairment. Deficits in performing an EDS are found in animals with a compromised prefrontal cortex, as has been shown both on the attentional set-shifting task (Birrell and Brown, 2000; McAlonan and Brown, 2003) and on similar radial maze-based tasks (Ragozzino et al, 1999a, b, 2003). Deficits on the set-shifting task have also been found following lesions of the parietal cortex (Fox et al, 2003), and, on a similar radial maze task, following interference with the medial dorsal ST (Palencia and Ragozzino, 2004; Ragozzino, 2003; Ragozzino et al, 2002a, b; Tzavos et al, 2004). MAM treatment could have impaired performance on the set-shifting task by altering functioning in one or all of these brain areas. The prefrontal cortex would seem a likely candidate since several previous reports have shown that MAM treatment at gestation day 17 disrupts prefrontal morphology and function (Flagstad et al, 2005; Lavin et al, 2005; Moore et al, 2006).

It is unclear as to whether difficulties in shifting attentional set are due to a failure to ignore a previously reinforced dimension or to attend to a previously irrelevant dimension. Lesions of the prefrontal cortex have been
Table 5 Brain Weights in Saline vs MAM-Treated Animals

\begin{tabular}{lcc}
\hline & Saline & MAM \\
\hline Hippocampus & I I $2.27(2.68)$ & $88.49(3.01)^{*}$ \\
Dorsal Striatum & II I.07 (2.24) & $96.62(5.00)^{*}$ \\
Nucleus accumbens & $9.92(0.62)$ & $8.65(0.39)$ \\
Medial prefrontal & $10.07(0.48)$ & $8.19(0.46)^{*}$ \\
Orbital prefrontal & $26.32(1.49)$ & $22.79(1.94)^{*}$ \\
Parietal & $239.16(6.54)$ & $187.07(7.16)^{*}$ \\
Amygdala & $53.17(1.32)$ & $50.42(1.15)$ \\
Thalamus & $125.86(2.94)$ & $123.32(9.08)$ \\
Hypothalamus & $37.91(1.49)$ & $39.22(1.94)$ \\
Cerebellum & $285.86(3.78)$ & $300.59(7.16)^{*}$ \\
\hline
\end{tabular}

Brain weights in selected regions in MAM-treated (MAM) vs saline-treated (SAL) animals. Asterisk $(*)$ indicates a significant $(p<0.05)$ difference in brain weight between MAM- and saline-treated animals. Aside from the cerebellum, MAMtreated animals had lower weights in all brain areas where significance was found.

shown to selectively increase perseveration to a previously learned dimension in non-human primates (Dias et al, 1996) and humans (Elliott et al, 1995; Owen et al, 1993). Given the likelihood that MAM disrupts prefrontal cortex function (Flagstad et al, 2004; Lavin et al, 2005) it would be expected that MAM-treated animals would likewise show increases in perseveration, and that this might serve as the 
basis for the deficit observed in the present study. In addition to an impairment in EDS learning, MAM-treated animals were impaired when required to make a simple reversal of a previously acquired discrimination (for two out of three reversals). Difficulties in reversal learning have been reported within a water maze paradigm in animals treated on gestational day 15 (Leng et al, 2005) and day 17 (Flagstad et al, 2005), as well as in a maze task in animals treated at day 17 (Moore et al, 2006). The finding of a reversal learning deficit in animals treated on gestational day 17 is important since treatment at this time leads to a much more restricted deficit, both behaviorally and anatomically (Gourevitch et al, 2004).

Previous work has shown that the prefrontal cortex plays a major role in several aspects of performance of the 5-choice task. Thus, prefrontal lesions alter accuracy (Chudasama et al, 2003; Passetti et al, 2002) and can induce changes in perseverative and premature responding (Chudasama et al, 2003; Murphy et al, 2005; Passetti et al, 2002). Given the effects of MAM treatment on attentional setshifting and reversal learning, as well as on prefrontal morphology, it is surprising that MAM treatment had little effect on the 5-choice performance. In the present study, MAM treatment did not disrupt accurate performance during any of the postacquisition manipulations (shortened stimulus durations, variable ITI and lengthened ITI), suggesting that performance of this task is not compromised by MAM treatment. Additionally, the lack of change in omitted trials and response latency suggests that attentional function was spared and, incidentally, indicates that motor and/or motivational functions were unaffected by MAM treatment. MAM-treated animals did show a trend towards an increase in premature responding during acquisition, suggesting a difficulty in behavioral inhibition. However, this change was nowhere near as significant as changes seen in premature responding following other prefrontal manipulations (Murphy et al, 2005). Although these two tasks would seem to be dependent upon a similar neuroanatomical substrate, the specific cognitive processes that underlie performance of each task are likely to be quite different. The set-shifting task requires animals to learn to attend to a particular feature of a complex stimulus, followed by a shift of attention away from that feature, two behaviors that could perhaps be best described as involving selective attention. In contrast, the 5-choice task requires animals to sustain attentional focus on a limited series of locations over a brief period of time, a process that could be described as involving attentional vigilance.

Given the suggestion of impaired response control on the 5-choice task, we sought to further assess MAM animals on a DRL schedule, a task that requires animals to space responses by at least $20 \mathrm{~s}$. MAM-treated animals showed profound disruptions in performance of this task, responding more frequently than saline-treated animals, earning fewer reinforcements, and displaying a M-IRT significantly shorter than what was required for optimal performance. Thus, by the end of training the maximal peak of responding in saline-treated animals occurred around $21 \mathrm{~s}$, while the peak level of responding in MAM-treated animals was around $16 \mathrm{~s}$. Interestingly, the two groups did not appear to differ in amount of burst responding (responses with $0-2 \mathrm{~s}$ IRTs). This result, coupled with the fact that the increase in overall responding in MAM-treated rats was relatively small $(\sim 5-10 \%)$ suggests that the deficits observed in MAM animals were not due to a generalized loss of behavioral inhibition. This overall pattern of results may be indicative of a deficit in interval timing, and might suggest that MAM-treated animals overestimate the passage of time, as has been shown to occur in schizophrenic patients (Elvevag et al, 2003, 2004; Penney et al, 2005). The finding of a deficit in DRL performance in MAM-treated animals is somewhat at odds with previous reports, which have shown a lack of deficit on this task following embryonic day 15 MAM treatment (Archer et al, 1988). It is possible that differences in procedure may explain these discrepancies or that the deficit observed on the current task may be specific to the disruption of developmental processes occurring around embryonic day 17.

In the present experiment, MAM treatment resulted in substantial decreases in brain tissue weight in the HI (21\% decrease in brain size in MAM-treated animals compared to saline-treated), medial prefrontal cortex (19\%), parietal cortex (22\%), dorsal ST (17\%), and the orbital prefrontal cortex (13\%), and these changes appear to be consistent with those observed elsewhere. For example, Flagstad et al (2004) reported brain weight decreases in the HI and prefrontal cortex of 17 and 22 percent, respectively.

The present study suggests that MAM may serve as a useful model of some aspects of schizophrenia. Superficially, the nature of brain change observed in MAM animals appears to be similar to that which occurs in schizophrenia. Additionally, the pattern of deficit observed in MAM animals on the attentional set-shifting task is in general agreement with what has been found in patients, although it is unclear whether this can be attributed to a disruption of similar cognitive processes as in schizophrenia or whether the similarities are more superficial. It is similarly unclear as to why MAM treatment did not affect performance of the 5-choice task and this result strongly suggests that MAM is not a suitable model of the types of deficits in sustained visual attention observed in this disease.

\section{ACKNOWLEDGEMENTS}

We thank John Chambers for performing brain dissections. REF was supported by a research fellowship from the Ontario Mental Health Foundation. SK is supported by a Canada Research Chair and by a Special Initiative grant from the Ontario Mental Health Foundation.

\section{REFERENCES}

Archer T, Hiltunen AJ, Jarbe TU, Kamkar MR, Luthman J, Sundstrom E et al (1988). Hyperactivity and instrumental learning deficits in methylazoxymethanol-treated rat offspring. Neurotoxicol Teratol 10: 341-347.

Birrell JM, Brown VJ (2000). Medial frontal cortex mediates perceptual attentional set shifting in the rat. J Neurosci 20: 4320 4324.

Brown VJ, Robbins TW (1989). Elementary processes of response selection mediated by distinct regions of the striatum. J Neurosci 9: $3760-3765$. 
Chen S, Hillman DE (1986). Selective ablation of neurons by methylazoxymethanol during pre- and postnatal brain development. Exp Neurol 94: 103-119.

Chen WJ, Faraone SV (2000). Sustained attention deficits as markers of genetic susceptibility to schizophrenia. Am J Med Genet 97: 52-57.

Christakou A, Robbins TW, Everitt BJ (2001). Functional disconnection of a prefrontal cortical-dorsal striatal system disrupts choice reaction time performance: implications for attentional function. Behav Neurosci 115: 812-825.

Chudasama Y, Passetti F, Rhodes SE, Lopian D, Desai A, Robbins TW (2003). Dissociable aspects of performance on the 5-choice serial reaction time task following lesions of the dorsal anterior cingulate, infralimbic and orbitofrontal cortex in the rat: differential effects on selectivity, impulsivity and compulsivity. Behav Brain Res 146: 105-119.

Dias R, Robbins TW, Roberts AC (1996). Primate analogue of the Wisconsin Card Sorting Test: effects of excitotoxic lesions of the prefrontal cortex in the marmoset. Behav Neurosci 110: 872-886.

Elliott R, McKenna PJ, Robbins TW, Sahakian BJ (1995). Neuropsychological evidence for frontostriatal dysfunction in schizophrenia. Psychol Med 25: 619-630.

Elvevag B, Brown GD, McCormack T, Vousden JI, Goldberg TE (2004). Identification of tone duration, line length, and letter position: an experimental approach to timing and working memory deficits in schizophrenia. J Abnorm Psychol 113: 509-521.

Elvevag B, McCormack T, Gilbert A, Brown GD, Weinberger DR, Goldberg TE (2003). Duration judgements in patients with schizophrenia. Psychol Med 33: 1249-1261.

Ferguson SA, Holson RR (1997). Methylazoxymethanol-induced micrencephaly in the brown Norway strain: behavior and brain weight. Int J Dev Neurosci 15: 75-86.

Fiore M, Aloe L, Westenbroek C, Amendola T, Antonelli A, Korf J (2001). Bromodeoxyuridine and methylazoxymethanol exposure during brain development affects behavior in rats: consideration for a role of nerve growth factor and brain derived neurotrophic factor. Neurosci Lett 309: 113-116.

Fiore M, Korf J, Antonelli A, Talamini L, Aloe L (2002). Longlasting effects of prenatal MAM treatment on water maze performance in rats: associations with altered brain development and neurotrophin levels. Neurotoxicol Teratol 24: 179-191.

Flagstad P, Glenthoj BY, Didriksen M (2005). Cognitive deficits caused by late gestational disruption of neurogenesis in rats: a preclinical model of schizophrenia. Neuropsychopharmacology 30: $250-260$.

Flagstad P, Mork A, Glenthoj BY, van Beek J, Michael-Titus AT, Didriksen M (2004). Disruption of neurogenesis on gestational day 17 in the rat causes behavioral changes relevant to positive and negative schizophrenia symptoms and alters amphetamineinduced dopamine release in nucleus accumbens. Neuropsychopharmacology 29: 2052-2064.

Fletcher PJ, Tenn CC, Rizos Z, Lovic V, Kapur S (2005). Sensitization to amphetamine, but not PCP, impairs attentional set shifting: reversal by a D1 receptor agonist injected into the medial prefrontal cortex. Psychopharmacology 183: 190-200.

Fox MT, Barense MD, Baxter MG (2003). Perceptual attentional set-shifting is impaired in rats with neurotoxic lesions of posterior parietal cortex. J Neurosci 23: 676-681.

Goldberg TE, Weinberger DR, Berman KF, Pliskin NH, Podd MH (1987). Further evidence for dementia of the prefrontal type in schizophrenia? A controlled study of teaching the Wisconsin Card Sorting Test. Arch Gen Psychiatry 44: 1008-1014.

Gourevitch R, Rocher C, Le Pen G, Krebs MO, Jay TM (2004). Working memory deficits in adult rats after prenatal disruption of neurogenesis. Behav Pharmacol 15: 287-292.

Grace A, Moore H (1998). Regulation of information flow in the nucleus accumbens: A model for the pathophysiology of schizophrenia. In: Lenzenweger MF, Dworkin RH (eds). Origins and Development of Schizophrenia: Advances in Experimental Psychopathology. American Psychological Association Press: Washington, DC. pp 123-157.

Green MF, Nuechterlein KH, Gold JM, Barch DM, Cohen J, Essock $S$ et al (2004). Approaching a consensus cognitive battery for clinical trials in schizophrenia: the NIMH-MATRICS conference to select cognitive domains and test criteria. Biol Psychiatry 56: 301-307.

Jongen-Relo AL, Leng A, Luber M, Pothuizen HH, Weber L, Feldon J (2004). The prenatal methylazoxymethanol acetate treatment: a neurodevelopmental animal model for schizophrenia? Behav Brain Res 149: 159-181.

Lavin A, Moore HM, Grace AA (2005). Prenatal disruption of neocortical development alters prefrontal cortical neuron responses to dopamine in adult rats. Neuropsychopharmacology 30: $1426-1435$.

Lee MH, Rabe A (1992). Premature decline in Morris water maze performance of aging micrencephalic rats. Neurotoxicol Teratol 14: $383-392$.

Leng A, Jongen-Relo AL, Pothuizen HH, Feldon J (2005). Effects of prenatal methylazoxymethanol acetate (MAM) treatment in rats on water maze performance. Behav Brain Res 161: 291-298.

Lu MH, Tang N, Ali SF (2000). Effects of single injection of methylazoxymethanol at postnatal day one on cell proliferation in different brain regions of male rats. Neurotoxicology 21: 11451151.

McAlonan K, Brown VJ (2003). Orbital prefrontal cortex mediates reversal learning and not attentional set shifting in the rat. Behav Brain Res 146: 97-103.

Mohammed AK, Jonsson G, Soderberg U, Archer T (1986a). Impaired selective attention in methylazoxymethanol-induced microencephalic rats. Pharmacol Biochem Behav 24: 975-981.

Mohammed AK, Jonsson G, Sundstrom E, Minor BG, Soderberg U, Archer T (1986b). Selective attention and place navigation in rats treated prenatally with methylazoxymethanol. Brain Res 395: $145-155$.

Moore H, Jentsch JD, Ghajarnia M, Geyer MA, Grace AA (2006). A neurobehavioral systems analysis of adult rats exposed to methylazoxymethanol acetate on E17: implications for the Neuropathology of schizophrenia. Biol Psychiatry 60: 253-264.

Muir JL, Everitt BJ, Robbins TW (1996). The cerebral cortex of the rat and visual attentional function: dissociable effects of mediofrontal, cingulate, anterior dorsolateral, and parietal cortex lesions on a five-choice serial reaction time task. Cereb Cortex 6: 470-481.

Murphy ER, Dalley JW, Robbins TW (2005). Local glutamate receptor antagonism in the rat prefrontal cortex disrupts response inhibition in a visuospatial attentional task. Psychopharmacology (Berlin) 179: 99-107.

Nuechterlein KH, Robbins TW, Einat H (2005). Distinguishing separable domains of cognition in human and animal studies: what separations are optimal for targeting interventions? A summary of recommendations from breakout group 2 at the measurement and treatment research to improve cognition in schizophrenia new approaches conference. Schizophr Bull 31: 870-874.

Owen AM, Roberts AC, Hodges JR, Summers BA, Polkey CE, Robbins TW (1993). Contrasting mechanisms of impaired attentional set-shifting in patients with frontal lobe damage or Parkinson's disease. Brain 116(Part 5): 1159-1175.

Palencia CA, Ragozzino ME (2004). The influence of NMDA receptors in the dorsomedial striatum on response reversal learning. Neurobiol Learn Mem 82: 81-89.

Passetti F, Chudasama Y, Robbins TW (2002). The frontal cortex of the rat and visual attentional performance: dissociable functions of distinct medial prefrontal subregions. Cereb Cortex 12: $1254-1268$. 
Penney TB, Meck WH, Roberts SA, Gibbon J, Erlenmeyer-Kimling L (2005). Interval-timing deficits in individuals at high risk for schizophrenia. Brain Cogn 58: 109-118.

Ragozzino ME (2003). Acetylcholine actions in the dorsomedial striatum support the flexible shifting of response patterns. Neurobiol Learn Mem 80: 257-267.

Ragozzino ME, Detrick S, Kesner RP (1999a). Involvement of the prelimbic-infralimbic areas of the rodent prefrontal cortex in behavioral flexibility for place and response learning. J Neurosci 19: 4585-4594.

Ragozzino ME, Jih J, Tzavos A (2002a). Involvement of the dorsomedial striatum in behavioral flexibility: role of muscarinic cholinergic receptors. Brain Res 953: 205-214.

Ragozzino ME, Kim J, Hassert D, Minniti N, Kiang C (2003). The contribution of the rat prelimbic-infralimbic areas to different forms of task switching. Behav Neurosci 117: 1054-1065.

Ragozzino ME, Ragozzino KE, Mizumori SJ, Kesner RP (2002b). Role of the dorsomedial striatum in behavioral flexibility for response and visual cue discrimination learning. Behav Neurosci 116: 105-115.

Ragozzino ME, Wilcox C, Raso M, Kesner RP (1999b). Involvement of rodent prefrontal cortex subregions in strategy switching. Behav Neurosci 113: 32-41.

Robbins TW (2002). The 5-choice serial reaction time task: behavioural pharmacology and functional neurochemistry. Psychopharmacology (Berlin) 163: 362-380.

Rogers RD, Baunez C, Everitt BJ, Robbins TW (2001). Lesions of the medial and lateral striatum in the rat produce differential deficits in attentional performance. Behav Neurosci 115: 799-811.

Shimizu J, Tamaru M, Katsukura T, Matsutani T, Nagata Y (1991). Effects of fetal treatment with methylazoxymethanol acetate on radial maze performance in rats. Neurosci Res 11: 209-214.

Talamini LM, Ellenbroek B, Koch T, Korf J (2000). Impaired sensory gating and attention in rats with developmental abnormalities of the mesocortex. Implications for schizophrenia. Ann NY Acad Sci 911: 486-494.

Talamini LM, Koch T, Luiten PG, Koolhaas JM, Korf J (1999). Interruptions of early cortical development affect limbic association areas and social behaviour in rats; possible relevance for neurodevelopmental disorders. Brain Res 847: 105-120.

Tzavos A, Jih J, Ragozzino ME (2004). Differential effects of M1 muscarinic receptor blockade and nicotinic receptor blockade in the dorsomedial striatum on response reversal learning. Behav Brain Res 154: 245-253.

Virgili M, Vandi M, Contestabile A (1997). Ischemic and excitotoxic damage to brain slices from normal and microencephalic rats. Neurosci Lett 233: 53-57. 